\title{
Functions with the one-dimensional holomorphic extension property
}

\author{
Simona G.Myslivets \\ Siberian Federal University, Krasnoyarsk, Russia
}

This paper presents some results related to the holomorphic extension of functions $f$, defined on the boundary of a domain $D \subset \mathbb{C}^{n}, n>1$, into this domain. It's about a functions with the one-dimensional holomorphic extension property along the complex lines.

The first result related to our subject was received M.L.Agranovsky and R.E.Valsky in [1], who studied the functions with a one-dimensional holomorphic continuation property into a ball. The proof was based on the automorphism group properties of a sphere.

E.L.Stout in [2] used complex Radon transformation to generalize the Agranovsky and Valsky theorem for an arbitrary bounded domain with a smooth boundary. An alternative proof of the Stout theorem was obtained by A.M.Kytmanov in [3] by applying the Bochner-Martinelli integral. The idea of using the integral representations (Bochner - Martinelli, Cauchy - Fantappiè, logarithmic residue) has been useful in the study the functions with one-dimensional holomorphic continuation property (see review [4]).

The question of finding the different families of complex lines, sufficient for holomorphic extension was put in [5]. As shown in [6], the family of complex lines passing through a finite number of points also, generally speaking, is not sufficient. Thus, a simple analogy theorem of Hartogs should be not expected.

Various other families are given in [7] - [11]. In [12] - [16] it is shown that for holomorphic extension of continuous functions defined on the boundary of the ball, enough $n+1$ points inside the ball, do not lie on a complex hyperplane. By the author and A.Kytmanov this result was generalized on the $n$-circular [17] and circular domains.

Now we formulate some results about the different sufficient families of complex lines for holomorphic extension.

Let $D$ be a bounded domain in $\mathbb{C}^{n}$ with a smooth boundary. Consider the complex line of the form

$$
l_{z, b}=\left\{\zeta \in \mathbb{C}^{n}: \zeta=z+b t, t \in \mathbb{C}\right\}=\left\{\left(\zeta_{1}, \ldots \zeta_{n}\right): \zeta_{j}=z_{j}+b_{j} t, j=1,2, \ldots, n, t \in \mathbb{C}\right\},
$$

where $z \in \mathbb{C}^{n}, b \in \mathbb{C P}^{n-1}$.

We will say that the function $f \in \mathcal{C}(\partial D)$ has the one-dimensional holomorphic extension property along the complex line $l_{z, b}$, if $\partial D \cap l_{z, b} \neq \varnothing$ and there exists a function $F_{l_{z, b}}$ with the following properties:

1) $F_{l_{z, b}} \in \mathcal{C}\left(\bar{B} \cap l_{z, b}\right)$,

2) $F_{l_{z, b}}=f$ on the set $\partial D \cap l_{z, b}$

3) function $F_{l_{z, b}}$ is holomorphic at the interior (with respect to the topology of $l_{z, b}$ ) points of set $\bar{D} \cap l_{z, b}$.

Let $\Gamma$ is a set in $\mathbb{C}^{n}$. Denote by $\mathfrak{L}_{\Gamma}$ the set of all complex lines $l_{z, b}$ such that $z \in \Gamma$, and $b \in \mathbb{C P}^{n-1}$, i.e., the set of all complex lines passing through $z \in \Gamma$. 
We will say that a function $f \in \mathcal{C}(\partial D)$ has the one-dimensional holomorphic extension property along the family $\mathfrak{L}_{\Gamma}$, if it has the one-dimensional holomorphic extension property along any complex line $l_{z, b} \in \mathfrak{L}_{\Gamma}$.

We will say set $\mathfrak{L}_{\Gamma}$ sufficient for holomorphic extension, if the function $f \in \mathcal{C}(\partial D)$ has the one-dimensional holomorphic extension property along all complex lines of the family $\mathfrak{L}_{\Gamma}$, and then the function $f$ extends holomorphically into $D$ (i.e., $f$ is a $C R$-function on $\partial D)$.

In what follows we will need the definition of a domain with the Nevanlinna property [18]. Let $G \subset \mathbb{C}$ be a simply connected domain and $t=k(\tau)$ be a conformal mapping of the unit circle $\Delta=\{\tau:|\tau|<1\}$ on $G$.

Domain $G$ is a domain with the Nevanlinna property, if there are bounded holomorphic functions $u$ and $v$ in $G$ such that almost everywhere on $S=\partial \Delta$, the equality

$$
\bar{k}(\tau)=\frac{u(k(\tau))}{v(k(\tau))}
$$

holds in terms of the angular boundary values. Essentially this means

$$
\bar{t}=\frac{u(t)}{v(t)} \quad \text { on } \quad \partial G .
$$

Give a characterization of a domains with the Nevanlinna property (Proposition 3.1 in [18]). Domain $G$ is a domain with the Nevanlinna property if and only if $k(\tau)$ admits a holomorphic pseudocontinuation through $S$ in $\overline{\mathbb{C}} \backslash \bar{\Delta}$, i.e., there are bounded holomorphic functions $u_{1}$ and $v_{1}$ such that the function $\tilde{k}(\tau)=\frac{u_{1}(\tau)}{v_{1}(\tau)}$ coincides almost everywhere with the function $k(\tau)$ on $S$.

The above definition and statement will be applied to bounded domains $G$ with a boundary of class $\mathcal{C}^{2}$, therefor (due to the principle of correspondence of boundaries) the function $k(\tau)$ extends to $\bar{\Delta}$ as a function of class $\mathcal{C}^{1}(\bar{\Delta})$ and $\tilde{k}(\tau)$ extends to $\mathbb{C} \backslash \bar{\Delta}$ as a function of class $\mathcal{C}(\mathbb{C} \backslash \bar{\Delta})$. Therefor in whole the function $\bar{t}=\frac{u(\tau)}{v(\tau)}$ is a meromorphic function in $\mathbb{C}$. Various example of domains with the Nevanlinna property are given in [18]. For example, if $\partial G$ is a real-analytic, then $k(\tau)$ is a rational function with no poles on the closure $\Delta$.

In our further consideration we will need the domain $G$ to possess the strengthened Nevanlinna property, that is the function $u_{1}(\tau) \neq 0$ in $\mathbb{C} \backslash \Delta$ and $\tilde{k}$ has at infinity zero of no more than first order. If $G=\Delta$ then $\bar{\tau}=\frac{1}{\tau}$ on $\partial \Delta$. Therefor meromorphic function $\frac{1}{\tau}$ has a zero of the first order at $\infty$.

For example, such domain will include domains for which $k(\tau)$ is a rational function with no poles on $\bar{\Delta}$ and no zeros in $\mathbb{C} \backslash \Delta$.

We will to say that the domain $D \subset \mathbb{C}^{n}$ possess the strengthened Nevanlinna property in the point $z \in D$ if the section $D \cap l_{z, b}$ possess the strengthened Nevanlinna property for any $b \in \mathbb{C} \mathbb{P}^{n-1}$. 
We formulate some results about the different families of complex lines sufficient for holomorphic extension.

Now we consider families of complex lines passing through a generic manifold. The real dimension of such a manifold is at least $n$. Recall that a smooth manifold $\Gamma$ of class $\mathcal{C}^{\infty}$ is said to be generic if the complex linear span of the tangent space $T_{z}(\Gamma)$ coincides with $\mathbb{C}^{n}$ for each point $z \in \Gamma$. We denote the family of all complex lines intersecting $\Gamma$ by $\mathfrak{L}_{\Gamma}$.

Theorem 1. Let $\Gamma$ be a germ of a generic manifold in $\mathbb{C}^{n} \backslash \bar{D}$ and the function $f \in \mathcal{C}(\partial D)$ have the one-dimensional holomorphic extension property along the family $\mathfrak{L}_{\Gamma}$, then the function $f$ extends holomorphically into the domain $D$.

Here we consider a generic manifold $\Gamma$ lying in the domain $D$.

Theorem 2. Let $\Gamma$ be a germ of a generic manifold in $D$ and a function $f \in \mathcal{C}(\partial D)$ have the one-dimensional holomorphic extension property along the family $\mathfrak{L}_{\Gamma}$ and the connected components of the intersection $D \cap l$ be domains with the strengthened Nevanlinna property, then the function $f$ extends holomorphically into the domain $D$.

Let $\Gamma$ be the germ of a complex manifold of dimension $(n-1)$ in $\mathbb{C}^{n}$, which lies outside $\bar{D}$. Having done the shift and unitary transformation, we can assume that $0 \in \Gamma, 0 \notin \bar{D}$ and that the complex hypersurface $\Gamma$ in some neighborhood $U$ of 0 has the form

$$
\Gamma=\left\{z \in U: z_{n}=\varphi\left(z^{\prime}\right), z^{\prime}=\left(z_{1}, \ldots, z_{n-1}\right)\right\},
$$

where $\varphi$ is the holomorphic function in a neighborhood of zero in $\mathbb{C}^{n-1}$ and $\varphi(0)=0$, $\frac{\partial \varphi}{\partial z_{k}}(0)=0, k=1, \ldots, n-1$.

We will assume that there is a direction $b^{0} \neq 0$ such that

$$
\left\langle b^{0}, \bar{\zeta}\right\rangle \neq 0 \quad \text { for all } \quad \zeta \in \bar{D} .
$$

Theorem 3. Let $D$ be a simply connected bounded domain and condition (1) be fulfilled and the function $f \in \mathcal{C}(\partial D)$ have the one-dimensional holomorphic extension property along the family $\mathfrak{L}_{\Gamma}$, then the function $f$ extends holomorphically into the domain $D$.

Let $B=\left\{z \in \mathbb{C}^{n}:|z|<1\right\}$ be a unit ball in $\mathbb{C}^{n}$ centered at the origin and let $S=\partial B$ be the boundary of the ball.

We denote by $\mathfrak{A}$ the set of points $a_{k} \in D \subset \mathbb{C}^{n}, k=1, \ldots, n+1$, which do not lie on the complex hyperplane in $\mathbb{C}^{n}$.

Theorem 4. Let a function $f \in \mathcal{C}(S)$ have the one-dimensional holomorphic extension property along the family $\mathfrak{L}_{\mathfrak{A}}$, then the function $f$ extends holomorphically into the ball $B$.

This theorem was proved for circular domains with the strengthened Nevanlinna property.

Theorem 5. Let $D$ be a bounded strictly convex circular domain with twice smooth boundary in $\mathbb{C}^{n}$ and possess the strengthened Nevanlinna property in the points from the set $\mathfrak{A}$ and a function $f \in \mathcal{C}(\partial D)$ have the one-dimensional holomorphic extension property along the family $\mathfrak{L}_{\mathfrak{A}}$, then the function $f$ extends holomorphically into the domain $D$. 


\section{References}

[1] M.L.Agranovsky and R.E.Valsky, Maximality of invariant algebras of functions, Sib. Mat. J. 12(1), 3-12 (1971).

[2] E.L.Stout, The boundary values of holomorphic functions of several complex variables, Duke Math. J. 44(1), 105-108 (1977).

[3] L.A.Aizenberg and A.P.Yuzhakov, Integral Representations and Residues in Multidimensional Complex Analysis, Tranclantions of Mathematical Monographs Vol. 58 (American Mathematical Society, Providence, RI, 1983).

[4] A.M.Kytmanov and S.G.Myslivets, Higher-dimensional boundary analogs of the Morera theorem in problems of analytic continuation of functions, J. Math. Sci. 120(6), 1842-1867 (2004).

[5] J.Globevnik and E.L.Stout, Boundary Morera theorems for holomorphic functions of several complex variables, Duke Math. J. 64(3), 571-615 (1991).

[6] A.M.Kytmanov and S.G.Myslivets, On the families of complex lines, sufficient for holomorphic continuations, Math. Notes, 83(4), 545 - 551 (2008).

[7] A.M.Kytmanov, S.G.Myslivets and V.I.Kuzovatov, Families of complex lines of the minimal dimension, sufficient for holomorphic continuation of functions, Sib. Math. J. 52(2), 256-266 (2011).

[8] M.Agranovsky, Propagation of boundary $C R$-foliations and Morera type theorems for manifolds with attached analytic discs, Advan. in Math. 211(1), 284-326 (2007).

[9] M.Agranovsky, Analog of a theorem of Forelli for boundary values of holomorphic functions on the unit ball of $\mathbb{C}^{n}$, J. d'Anal. Math. 13(1), 293-304 (2011).

[10] L.Baracco, Holomorphic extension from the sphere to the ball, J. Math. Anal. Appl. 388(2), 760-762 (2012).

[11] J.Globevnik, Small families of complex lines for testing holomorphic extendibility, Am. J. Math. 134(6), 1473-1490 (2012).

[12] L.Baracco, Separate holomorphic extension along lines and holomorphic extension from the sphere to the ball, Am. J. Math. 135(2), 493-497 (2013).

[13] J.Globevnik, Meromorphic extensions from small families of circles and holomorphic extensions from spheres, Trans. Am. Math. Soc. 364(11), 5857-5880 (2012).

[14] A.M.Kytmanov and S.G.Myslivets, Holomorphic extension of functions along finite families of complex linea in a ball, J. Sib. Fed. Univ. Math. and Phys. 5(4), 547-557 (2012). 
[15] A.M.Kytmanov and S.G.Myslivets, An analog of the Hartogs theorem in a ball of $\mathbb{C}^{n}$, Math. Nahr. 288(2-3), 224-234 (2015).

[16] A.M.Kytmanov and S.G.Myslivets, Multidimensional Integral Representations (Springer Inter. Publ. Switzarland, 2015).

[17] A.M.Kytmanov and S.G.Myslivets, Holomorphic extension of functions along finite families of complex linea in a $n$-circular domain, Sib. Math. J. 57(4), 618-631 (2016).

[18] J.J.Carmona, P.V.Paramonov, K.Yu.Fedorovskii, On uniform approximation by polyanalytic polynomials and the Dirichlet problem for bianalytic functions, Sb. Math. 193, 1469-1492 (2002). 